\title{
Stochastic integration based on simple, symmetric random walks
}

\author{
Tamás Szabados*† and Balázs Székely $\ddagger$ \\ Budapest University of Technology and Economics
}

\begin{abstract}
A new approach to stochastic integration is described, which is based on an a.s. pathwise approximation of the integrator by simple, symmetric random walks. Hopefully, this method is didactically more advantageous, more transparent, and technically less demanding than other existing ones. In a large part of the theory one has a.s. uniform convergence on compacts. In particular, it gives a.s. convergence for the stochastic integral of a finite variation function of the integrator, which is not càdlàg in general.
\end{abstract}

\section{Introduction}

The main purpose of the present paper is to describe a new approach to stochastic integration, which is based on an a.s. pathwise approximation of the integrator by simple, symmetric random walks. It is hoped that this method is pedagogically more advantageous, more transparent, and technically less demanding than other existing ones. This way hopefully a larger, mathematically less mature audience may get acquainted with a theory of stochastic integration. Since stochastic calculus plays an ever-increasing role in several applications (mathematical finance, statistical mechanics, engineering, ...) nowadays, this aim seems to be justified.

A basic feature of the present theory that, except for the integral of general predictable integrands, one always has almost sure uniform convergence on compact intervals. This is true for the approximation of the integrators, quadratic variations, local times, and stochastic integrals when the integrand is a $C^{1}$ or a finite variation function of the integrator. We believe that for a beginner it is easier to understand almost sure pathwise convergence than $L^{2}$ or in probability

\footnotetext{
*Corresponding author, address: Department of Mathematics, Budapest University of Technology and Economics, Müegyetem rkp. 3, H ép. V em. Budapest, 1521, Hungary, e-mail: szabados@math.bme.hu, telephone: (+36 1) 463-1111/ext. 5907, fax: (+36 1) 4631677

${ }^{\dagger}$ Research supported by a Hungarian National Research Foundation (OTKA) grant No. T42496.

${ }^{\ddagger}$ Research supported by the HSN laboratory of BUTE.

2000 MSC. Primary 60H05. Secondary 60F15, 60G50.

Keywords and phrases. Stochastic integration, strong approximation, random walk, Ito formula.
} 
convergence that typically appear in stochastic calculus textbooks. We mention that our method significantly differs from the a.s. converging approximation given by Karandikar 4. Important tools in our approach are discrete versions of Itô's and Itô-Tanaka formulas. The continuous versions easily follow from these by a.s. pathwise convergence. (See earlier versions of it in [9] and [10].) To our best knowledge, our approach is new in giving a.s. convergence for the stochastic integral of a finite variation function of the integrator, which is not càdlàg in general.

In the case of a more general, e.g. predictable integrand, our method is closer to the traditional ones, e.g. we too have $L^{2}$ convergence then. The only, hopefully interesting, difference is that in the approximating sums random partitions are used so that the values of the integrand are multiplied by \pm 1 times a constant scaling factor. The signs are from an independent, symmetric coin tossing sequence.

The most general integrators considered in this paper are continuous local martingales $M$. It is easy to extend this to semimartingales $X$ that can be written $X=M+A$, where $M$ is a continuous local martingale and $A$ is a finite variation process. The reason is simple: integration with respect to such an $A$ can also be defined pathwise.

\section{Preliminaries}

A basic tool of the present paper is an elementary construction of Brownian motion (BM). The specific construction we are going to use in the sequel, taken from [10, is based on a nested sequence of simple, symmetric random walks (RW's) that uniformly converges to the Wiener process $(=\mathrm{BM})$ on bounded intervals with probability 1. This will be called "twist and shrink" construction in the sequel. Our method is a modification of the one given by Frank Knight in 1962 [5.

We summarize the major steps of the "twist and shrink" construction here. We start with an infinite matrix of independent and identically distributed random variables $X_{m}(k), \mathbb{P}\left\{X_{m}(k)= \pm 1\right\}=\frac{1}{2}(m \geq 0, k \geq 1)$, defined on the same complete probability space $(\Omega, \mathcal{F}, \mathbb{P})$. (All stochastic processes in the sequel will be defined on this probability space.) Each row of this matrix is a basis of an approximation of the Wiener process with a dyadic step size $\Delta t=2^{-2 m}$ in time and a corresponding step size $\Delta x=2^{-m}$ in space. Thus we start with a sequence of independent simple, symmetric RW's $S_{m}(0)=0, S_{m}(n)=\sum_{k=1}^{n} X_{m}(k)$ $(n \geq 1)$.

The second step of the construction is twisting. From the independent RW's we want to create dependent ones so that after shrinking temporal and spatial step sizes, each consecutive RW becomes a refinement of the previous one. Since the spatial unit will be halved at each consecutive row, we define stopping times by $T_{m}(0)=0$, and for $k \geq 0$,

$$
T_{m}(k+1)=\min \left\{n: n>T_{m}(k),\left|S_{m}(n)-S_{m}\left(T_{m}(k)\right)\right|=2\right\} \quad(m \geq 1)
$$

These are the random time instants when a RW visits even integers, different from the previous one. After shrinking the spatial unit by half, a suitable modification of this RW will visit the same integers in the same order as the previous RW. We operate here on each point $\omega \in \Omega$ of the sample space separately, i.e. 
we fix a sample path of each RW. We define twisted RW's $\tilde{S}_{m}$ recursively for $k=1,2, \ldots$ using $\tilde{S}_{m-1}$, starting with $\tilde{S}_{0}(n)=S_{0}(n)(n \geq 0)$ and $\tilde{S}_{m}(0)=0$ for any $m \geq 0$. With each fixed $m$ we proceed for $k=0,1,2, \ldots$ successively, and for every $n$ in the corresponding bridge, $T_{m}(k)<n \leq T_{m}(k+1)$. Any bridge is flipped if its sign differs from the desired:

$$
\tilde{X}_{m}(n)=\left\{\begin{aligned}
X_{m}(n) & \text { if } S_{m}\left(T_{m}(k+1)\right)-S_{m}\left(T_{m}(k)\right)=2 \tilde{X}_{m-1}(k+1), \\
-X_{m}(n) & \text { otherwise, }
\end{aligned}\right.
$$

and then $\tilde{S}_{m}(n)=\tilde{S}_{m}(n-1)+\tilde{X}_{m}(n)$. Then $\tilde{S}_{m}(n)(n \geq 0)$ is still a simple symmetric RW [10, Lemma 1]. The twisted RW's have the desired refinement property:

$$
\tilde{S}_{m+1}\left(T_{m+1}(k)\right)=2 \tilde{S}_{m}(k) \quad(m \geq 0, k \geq 0) .
$$

The third step of the RW construction is shrinking. The sample paths of $\tilde{S}_{m}(n)(n \geq 0)$ can be extended to continuous functions by linear interpolation, this way one gets $\tilde{S}_{m}(t)(t \geq 0)$ for real $t$. The $m t h$ "twist and shrink" $R W$ is defined by

$$
\tilde{B}_{m}(t)=2^{-m} \tilde{S}_{m}\left(t 2^{2 m}\right) .
$$

Then the refinement property takes the form

$$
\tilde{B}_{m+1}\left(T_{m+1}(k) 2^{-2(m+1)}\right)=\tilde{B}_{m}\left(k 2^{-2 m}\right) \quad(m \geq 0, k \geq 0) .
$$

Note that a refinement takes the same dyadic values in the same order as the previous shrunken walk, but there is a time lag in general:

$$
T_{m+1}(k) 2^{-2(m+1)}-k 2^{-2 m} \neq 0 .
$$

It is clear that this construction is especially useful for local times, since a refinement approximates the local time of the previous walk, with a geometrically distributed random number of visits with half-length steps (cf. Theorem C below).

Now we quote some important facts from [10] and [12] about the above RW construction that will be used in the sequel.

Theorem A. On bounded intervals the sequence $\left(\tilde{B}_{m}\right)$ almost surely uniformly converges as $m \rightarrow \infty$ and the limit process is Brownian motion $W$. For any $C>1$, and for any $K>0$ and $m \geq 1$ such that $K 2^{2 m} \geq N(C)$, we have

$$
\begin{array}{r}
\mathbb{P}\left\{\sup _{0 \leq t \leq K}\left|W(t)-\tilde{B}_{m}(t)\right| \geq 27 C K_{*}^{\frac{1}{4}}\left(\log _{*} K\right)^{\frac{3}{4}} m^{\frac{3}{4}} 2^{-\frac{m}{2}}\right\} \\
\leq \frac{6}{1-4^{1-C}}\left(K 2^{2 m}\right)^{1-C}
\end{array}
$$

where $K_{*}:=K \vee 1$ and $\log _{*} K:=(\log K) \vee 1$.

$(N(C)$ here and in the sequel denotes a large enough integer depending on $C$, whose value can be different at each occasion.)

Conversely, with a given Wiener process $W$, one can define the stopping times which yield the Skorohod embedded $R W^{\prime} s B_{m}\left(k 2^{-2 m}\right)$ into $W$. For every $m \geq 0$ let $s_{m}(0)=0$ and

$$
s_{m}(k+1)=\inf \left\{s: s>s_{m}(k),\left|W(s)-W\left(s_{m}(k)\right)\right|=2^{-m}\right\} \quad(k \geq 0) .
$$


With these stopping times the embedded dyadic walks by definition are

$$
B_{m}\left(k 2^{-2 m}\right)=W\left(s_{m}(k)\right) \quad(m \geq 0, k \geq 0) .
$$

This definition of $B_{m}$ can be extended to any real $t \geq 0$ by pathwise linear interpolation.

If a Wiener process is built by the "twist and shrink" construction described above using a sequence $\left(\tilde{B}_{m}\right)$ of nested RW's and then one constructs the Skorohod embedded RW's $\left(B_{m}\right)$, it is natural to ask about their relationship. The next lemma explains that they are asymptotically identical. In general, roughly saying, $\left(\tilde{B}_{m}\right)$ is more useful when someone wants to generate stochastic processes from scratch, while $\left(B_{m}\right)$ is more advantageous when someone needs discrete approximations of given processes.

Lemma A. For any $C>1$, and for any $K>0$ and $m \geq 1$ such that $K 2^{2 m} \geq$ $N(C)$ take the following subset of $\Omega$ :

$$
A_{K, m}^{*}=\left\{\sup _{n>m} \sup _{k}\left|2^{-2 n} T_{m, n}(k)-k 2^{-2 m}\right|<\left(42 C K \log _{*} K\right)^{\frac{1}{2}} m^{\frac{1}{2}} 2^{-m}\right\},
$$

where $T_{m, n}(k)=T_{n} \circ T_{n-1} \circ \cdots \circ T_{m}(k)$ for $n>m \geq 0$ and $k \in\left[0, K 2^{2 m}\right]$. Then

$$
\mathbb{P}\left\{\left(A_{K, m}^{*}\right)^{c}\right\} \leq \frac{2}{1-4^{1-C}}\left(K 2^{2 m}\right)^{1-C} .
$$

Moreover, $\lim _{n \rightarrow \infty} 2^{-2 n} T_{m, n}(k)=t_{m}(k)$ exists almost surely and on $A_{K, m}^{*}$ we have

$$
\tilde{B}_{m}\left(k 2^{-2 m}\right)=W\left(t_{m}(k)\right) \quad\left(0 \leq k 2^{-2 m} \leq K\right),
$$

cf. (4). Further, almost everywhere on $A_{K, m}^{*}, s_{m}(k)=t_{m}(k)$ and

$$
\sup _{0 \leq k 2^{-2 m} \leq K}\left|s_{m}(k)-k 2^{-2 m}\right| \leq\left(42 C K \log _{*} K\right)^{\frac{1}{2}} m^{\frac{1}{2}} 2^{-m} .
$$

The next theorem shows that the rate of convergence of $\left(B_{m}\right)$ to $W$ is essentially the same as the one of $\left(\tilde{B}_{m}\right)$, cf. Theorem $\mathrm{A}$.

Theorem B. For any $C>1$, and for any $K>0$ and $m \geq 1$ such that $K 2^{2 m} \geq N(C)$ we have

$$
\begin{array}{r}
\mathbb{P}\left\{\sup _{0 \leq t \leq K}\left|W(t)-B_{m}(t)\right| \geq 27 C K_{*}^{\frac{1}{4}}\left(\log _{*} K\right)^{\frac{3}{4}} m^{\frac{3}{4}} 2^{-\frac{m}{2}}\right\} \\
\leq \frac{8}{1-4^{1-C}}\left(K 2^{2 m}\right)^{1-C} .
\end{array}
$$

In the last part of this section we quote a result from 12 about an elementary definition of Brownian local time, based on the "twist and shrink" RW's $\left(\tilde{B}_{m}(t)\right)$. This is basically a finite-time-horizon version of a theorem of Révész [7, in a somewhat sharper form.

First, one can define one-sided, up and down local times $\tilde{\ell}_{m}^{+}(k, x)$ and $\tilde{\ell}_{m}^{-}(k, x)$ of the "twisted" RW's $\left(\tilde{S}_{m}(j)\right)$ at a point $x \in \mathbb{Z}$ up to time $k \in \mathbb{Z}_{+}$as $\tilde{\ell}_{m}^{ \pm}(0, x)=$ 0 and

$$
\tilde{\ell}_{m}^{ \pm}(k, x)=\#\left\{j: 0 \leq j<k, \tilde{S}_{m}(j)=x, \tilde{S}_{m}(j+1)=x \pm 1\right\} \quad(k \geq 1) .
$$


Then the two-sided local time is $\tilde{\ell}_{m}(k, x)=\tilde{\ell}_{m}^{+}(k, x)+\tilde{\ell}_{m}^{-}(k, x)$.

The one-sided (and two-sided) local times of the $m$ th "twist and shrink" walk $\tilde{B}_{m}$ at a point $x \in 2^{-m} \mathbb{Z}$ up to time $t \in 2^{-2 m} \mathbb{Z}_{+}$are defined as

$$
\tilde{\mathcal{L}}_{m}^{ \pm}(t, x)=2^{-m} \tilde{\ell}_{m}^{ \pm}\left(t 2^{2 m}, x 2^{m}\right)
$$

corresponding to the fact that the spatial step size of $\tilde{B}_{m}$ is $2^{-m}$. Then $\tilde{\mathcal{L}}_{m}^{ \pm}(t, x)$ can be extended to any $t \in \mathbb{R}_{+}$and $x \in \mathbb{R}$ by linear interpolation, as a continuous two-parameter process.

Theorem C. On any strip $[0, K] \times \mathbb{R}$ the sequence $\left(\tilde{\mathcal{L}}_{m}^{+}(t, x)\right)$ almost surely uniformly converges as $m \rightarrow \infty$ to the one half of the Brownian local time $\mathcal{L}(t, x)$. For any $C>1$, and for any $K>0$ and $m \geq 1$ such that $K 2^{2 m} \geq N(C)$, we have

$$
\begin{array}{r}
\mathbb{P}\left\{\sup _{(t, x) \in[0, K] \times \mathbb{R}}\left|\frac{1}{2} \mathcal{L}(t, x)-\tilde{\mathcal{L}}_{m+1}^{+}(t, x)\right| \geq 50 C K_{*}^{\frac{1}{4}}\left(\log _{*} K\right)^{\frac{3}{4}} m^{\frac{3}{4}} 2^{-\frac{m}{2}}\right\} \\
\leq \frac{30}{1-4^{1-C}}\left(K 2^{2 m}\right)^{1-C} .
\end{array}
$$

Similar statements hold for $\left(\tilde{\mathcal{L}}_{m}^{-}(t, x)\right)$ as well.

Very similar statements hold when the "twist and shrink" walks $\tilde{B}_{m}(t)$ are replaced by Skorohod embedded walks $B_{m}(t)$ in the definition (5) and (6) of local time. This local time will be denoted by $\mathcal{L}_{m}^{ \pm}(t, x)$ in the sequel.

Corollary 1. On any strip $[0, K] \times \mathbb{R}$ the sequence $\left(\mathcal{L}_{m}^{+}(t, x)\right)$ almost surely uniformly converges as $m \rightarrow \infty$ to the one half of the Brownian local time $\mathcal{L}(t, x)$. For any $C>1$, and for any $K>0$ and $m \geq 1$ such that $K 2^{2 m} \geq N(C)$, we have

$$
\begin{array}{r}
\mathbb{P}\left\{\sup _{(t, x) \in[0, K] \times \mathbb{R}}\left|\frac{1}{2} \mathcal{L}(t, x)-\mathcal{L}_{m+1}^{+}(t, x)\right| \geq 50 C K_{*}^{\frac{1}{4}}\left(\log _{*} K\right)^{\frac{3}{4}} m^{\frac{3}{4}} 2^{-\frac{m}{2}}\right\} \\
\leq \frac{32}{1-4^{1-C}}\left(K 2^{2 m}\right)^{1-C} .
\end{array}
$$

Similar statements hold for $\left(\mathcal{L}_{m}^{-}(t, x)\right)$ as well.

Proof. By Lemma $\mathrm{A}$, almost everywhere on an event $A_{K, m}^{*}$ whose complement has probability going to zero exponentially fast with $m$, one has that

$$
\tilde{B}_{m}\left(k 2^{-2 m}\right)=W\left(t_{m}(k)\right)=W\left(s_{m}(k)\right)=B_{m}\left(k 2^{-2 m}\right) \quad\left(0 \leq k 2^{-2 m} \leq K\right) .
$$

Thus on $A_{K, m}^{*}$, for almost every $\omega$ and for all $(t, x) \in[0, K] \times \mathbb{R}, \tilde{\mathcal{L}}_{m}^{+}(t, x)=$ $\mathcal{L}_{m}^{+}(t, x)$.

Hence, by the triangle inequality

$$
\sup _{(t, x)}\left|\mathcal{L}^{+}(t, x)-\mathcal{L}_{m}^{+}(t, x)\right| \leq \sup _{(t, x)}\left|\tilde{\mathcal{L}}_{m}^{+}(t, x)-\mathcal{L}_{m}^{+}(t, x)\right|+\sup _{(t, x)}\left|\mathcal{L}^{+}(t, x)-\tilde{\mathcal{L}}_{m}^{+}(t, x)\right|,
$$


and by Theorem $\mathrm{C}$, we obtain the above statement:

$$
\begin{aligned}
\mathbb{P} & \left\{\sup _{(t, x) \in[0, K] \times \mathbb{R}}\left|\frac{1}{2} \mathcal{L}(t, x)-\mathcal{L}_{m+1}^{+}(t, x)\right| \geq 50 C K_{*}^{\frac{1}{4}}\left(\log _{*} K\right)^{\frac{3}{4}} m^{\frac{3}{4}} 2^{-\frac{m}{2}}\right\} \\
& \leq \mathbb{P}\left\{\left(A_{K, m}^{*}\right)^{c}\right\} \\
& +\mathbb{P}\left\{\sup _{(t, x) \in[0, K] \times \mathbb{R}}\left|\frac{1}{2} \mathcal{L}(t, x)-\tilde{\mathcal{L}}_{m+1}^{+}(t, x)\right| \geq 50 C K_{*}^{\frac{1}{4}}\left(\log _{*} K\right)^{\frac{3}{4}} m^{\frac{3}{4}} 2^{-\frac{m}{2}}\right\} \\
& \leq \frac{32}{1-4^{1-C}}\left(K 2^{2 m}\right)^{1-C} .
\end{aligned}
$$

\section{A discrete Itô-Tanaka formula and its conse- quences}

It is interesting that one can give discrete versions of Itô's formula and of ItôTanaka formula, which are purely algebraic identities, not assigning any probabilities to the terms. Despite this, the usual Itô's and Itô-Tanaka formulae follow fairly easily in a proper probability setting.

Discrete Itô formulas are not new. Apparently, the first such formula was given by Kudzma in 1982 6]. A similar approach was recently used by Fujita [3] and Akahori [1. Discrete Tanaka formulae were given by Kudzma 6] and Csörgo"-Révész [2]. The elementary algebraic approach used in the present paper is different from these; it was introduced by the first author in 1989 [9].

First consider an arbitrary numerical sequence $X_{r}= \pm 1(r \geq 1)$ and partial sums $S_{0}=a \in \mathbb{R}, S_{n}=a+\left(X_{1}+\cdots+X_{n}\right) \Delta x(n \geq 1)$, where $\Delta x>0$ is the step-size. Let $f: \mathbb{R} \rightarrow \mathbb{R}$, and $b=a+k \Delta x, k \in \mathbb{Z}$. We define trapezoidal sums of $f$ with step $\Delta x$ on the interval $[a, b]$ by

$$
T_{x=a}^{b} f(x) \Delta x:=\operatorname{sgn}(b-a) \Delta x\left\{\frac{1}{2} f(a)+\sum_{j=1}^{|k|-1} f(a+\operatorname{sgn}(b-a) j \Delta x)+\frac{1}{2} f(b)\right\}
$$

where $\operatorname{sgn}(x)=1,0,-1$ according as $x>0, x=0$, or $x<0$, respectively.

Similarly as above, one-sided, up and down local times $\mathcal{L}^{+}(n, x)$ and $\mathcal{L}^{-}(n, x)$ $(n \geq 0)$ of the series $\left(S_{n}\right)$ can be defined with step $\Delta x: \mathcal{L}^{ \pm}(0, x):=0$ and

$$
\mathcal{L}^{ \pm}(n, x):=\Delta x \#\left\{j: 0 \leq j<n, S_{j}=x, S_{j+1}=x \pm \Delta x\right\}
$$

where $n \geq 1$ and $x=a+k \Delta x, k \in \mathbb{Z}$. The two-sided local time is $\mathcal{L}(n, x):=$ $\mathcal{L}^{+}(n, x)+\mathcal{L}^{-}(n, x)$.

Lemma 1. Take any function $f: \mathbb{R} \rightarrow \mathbb{R}, a \in \mathbb{R}$, step $\Delta x>0$, and numerical sequence $X_{r}= \pm 1(r \geq 1)$. Let $S_{0}=a, S_{n}=a+\left(X_{1}+\cdots+X_{n}\right) \Delta x(n \geq 1)$. Then the following equalities hold:

$$
T_{x=S_{0}}^{S_{n}} f(x) \Delta x=\sum_{r=1}^{n} \frac{f\left(S_{r}\right)+f\left(S_{r-1}\right)}{2} X_{r} \Delta x
$$


(discrete Stratonovich formula). Alternatively,

$$
T_{x=S_{0}}^{S_{n}} f(x) \Delta x=\sum_{r=1}^{n} f\left(S_{r-1}\right) X_{r} \Delta x+\frac{1}{2} \sum_{r=1}^{n} \frac{f\left(S_{r}\right)-f\left(S_{r-1}\right)}{X_{r} \Delta x}(\Delta x)^{2}
$$

(discrete Itô's formula). Finally,

$$
\begin{aligned}
& T_{x=S_{0}}^{S_{n}} f(x) \Delta x=\sum_{r=1}^{n} f\left(S_{r-1}\right) X_{r} \Delta x \\
& \quad+\frac{1}{2} \sum_{x \in a+\mathbb{Z} \Delta x}\left\{\mathcal{L}^{+}(n, x)(f(x+\Delta x)-f(x))+\mathcal{L}^{-}(n, x)(f(x)-f(x-\Delta x))\right\}
\end{aligned}
$$

(discrete Itô-Tanaka formula).

Proof. Algebraically,

$$
\begin{array}{rl}
T_{x=S_{0}}^{S_{r}} & f(x) \Delta x-T_{x=S_{0}}^{S_{r-1}} f(x) \Delta x=X_{r} \Delta x \frac{f\left(S_{r-1}\right)+f\left(S_{r}\right)}{2} \\
= & f\left(S_{r-1}\right) X_{r} \Delta x+\frac{1}{2} \frac{f\left(S_{r}\right)-f\left(S_{r-1}\right)}{X_{r} \Delta x}(\Delta x)^{2} \\
= & f\left(S_{r-1}\right) X_{r} \Delta x \\
& +\frac{1}{2} \Delta x \sum_{x \in a+\mathbb{Z} \Delta x}(f(x+\Delta x)-f(x)) \mathbf{1}_{\left\{S_{r-1}=x, S_{r}=x+\Delta x\right\}} \\
& +\frac{1}{2} \Delta x \sum_{x \in a+\mathbb{Z} \Delta x}(f(x)-f(x-\Delta x)) \mathbf{1}_{\left\{S_{r-1}=x, S_{r}=x-\Delta x\right\}} .
\end{array}
$$

The first equality follows from the fact that if $X_{r}=1$, one has to add a term, while if $X_{r}=-1$, one has to subtract a term in the trapezoidal sum. Then the second equality follows since $1 / X_{r}=X_{r}$, and the third equality is obviously implied by the second. Summing up for $r=1, \ldots, n$, the sum on the left telescopes, and from the three equalities one obtains the three formulae, respectively.

Introducing the notation $h_{ \pm \Delta x}(x):=(f(x \pm \Delta x)-f(x)) /( \pm \Delta x)$ and comparing (8) and (9), we obtain a discrete occupation time formula, cf. (19):

$$
\sum_{r=1}^{n} h_{X_{r} \Delta x}\left(S_{r-1}\right)(\Delta x)^{2}=\sum_{x \in a+\mathbb{Z} \Delta x}\left\{h_{\Delta x}(x) \mathcal{L}^{+}(n, x)+h_{-\Delta x}(x) \mathcal{L}^{-}(n, x)\right\} \Delta x .
$$

Let us apply Lemma 1 with Skorohod embedded walks $\left(B_{m}(t)\right)$. Elementary calculus shows, c.f. [9, Theorem 6], that when $g \in C^{2}(\mathbb{R})$ and we set $f=$ $g^{\prime}$ in (7) or in (8), then, as $m \rightarrow \infty$, the terms converge almost surely to the corresponding terms of the Stratonovich and Itô's formula, respectively. On one hand, this gives a definition of the Itô integral $\int_{0}^{t} f(W(s)) \mathrm{d} W(s)$ and Stratonovich integral $\int_{0}^{t} f(W(s)) \partial W(s)$, with almost sure convergence of Itô sums

$$
(f(W) \cdot W)_{t}^{m}:=\sum_{r=1}^{\left\lfloor t 2^{2 m}\right\rfloor} f\left(B_{m}\left((r-1) 2^{-2 m}\right)\right) 2^{-m} X_{m}(r)
$$


and Stratonovich sums

$$
(f(W) \circ W)_{t}^{m}:=\sum_{r=1}^{\left\lfloor t 2^{2 m}\right\rfloor} \frac{f\left(B_{m}\left((r-1) 2^{-2 m}\right)\right)+f\left(B_{m}\left(r 2^{-2 m}\right)\right)}{2} 2^{-m} X_{m}(r)
$$

respectively. Here $\left(X_{m}(r)\right)_{r=1}^{\infty}$ is an independent, \pm 1 symmetric coin tossing sequence, $2^{-m} X_{m}(r)=B_{m}\left(r 2^{-2 m}\right)-B_{m}\left((r-1) 2^{-2 m}\right)$. This essentially means that in the sums approximating the Itô integral we apply a partition with the Skorohod stopping times $0=s_{m}(0)<s_{m}(1)<\cdots<s_{m}\left(\left\lfloor t 2^{2 m}\right\rfloor\right)$, since $B_{m}\left(r 2^{-2 m}\right)=W\left(s_{m}(r)\right)$. Second, taking almost sure limits as $m \rightarrow \infty$, one immediately obtains the corresponding Itô's formula

$$
g(W(t))-g(W(0))=\int_{0}^{t} g^{\prime}(W(s)) \mathrm{d} W(s)+\frac{1}{2} \int_{0}^{t} g^{\prime \prime}(W(s)) \mathrm{d} s,
$$

and Stratonovich formula

$$
g(W(t))-g(W(0))=\int_{0}^{t} g^{\prime}(W(s)) \partial W(s) .
$$

In the same way, now we show almost sure convergence of stochastic sums $(f(W) \cdot W)_{t}^{m}$, when $g$ is the difference of two convex functions and $f=g_{-}^{\prime}$, its left derivative. Then we immediately obtain the Itô-Tanaka formula as well, with the help of (9).

Theorem 1. Let $g$ be the difference of two convex functions, $g_{-}^{\prime}$ be its left derivative, and let $\mu$ be the signed measure defined by the change of $g_{-}^{\prime}$ when restricted to compacts (the second derivative of $g$ in the generalized function sense). Then for arbitrary $K>0$,

$$
\sup _{t \in[0, K]}\left|\left(g_{-}^{\prime}(W) \cdot W\right)_{t}^{m}-\int_{0}^{t} g_{-}^{\prime}(W(s)) \mathrm{d} W(s)\right| \rightarrow 0
$$

almost surely as $m \rightarrow \infty$, and for any $t \geq 0$,

$$
g(W(t))-g(W(0))=\int_{0}^{t} g_{-}^{\prime}(W(s)) \mathrm{d} W(s)+\frac{1}{2} \int_{-\infty}^{\infty} \mathcal{L}(t, x) \mu(\mathrm{d} x) .
$$

Proof. The basic idea of the proof is that one may substitute Skorohod embedded walks $B_{m}\left(r 2^{-2 m}\right)=W\left(s_{m}(r)\right), B_{m}(0)=W(0)=a \in \mathbb{R}$ into (9) to obtain

$$
\begin{aligned}
\left(g_{-}^{\prime}(W) \cdot W\right)_{t}^{m} & =T_{x=a}^{B_{m}\left(\left\lfloor t 2^{2 m}\right\rfloor 2^{-2 m}\right)} g_{-}^{\prime}(x) 2^{-m} \\
& -\frac{1}{2} \sum_{x \in a+2^{-m} \mathbb{Z}}\left\{\mathcal{L}_{m}^{+}(t, x)\left(g_{-}^{\prime}\left(x+2^{-m}\right)-g_{-}^{\prime}(x)\right)\right. \\
& \left.\quad+\mathcal{L}_{m}^{-}(t, x)\left(g_{-}^{\prime}(x)-g_{-}^{\prime}\left(x-2^{-m}\right)\right)\right\} .
\end{aligned}
$$

Then it is enough to deal with the almost sure uniform convergence on compacts of the terms on the right side of (14), and that will imply the same convergence of the stochastic sum on the left side and will prove the Itô-Tanaka formula (13) as well. 
First we show (13) for measures $\mu$ supported in a compact interval $[-M, M]$. Because of the linearity of (13), it can be supposed that $g$ is convex. Then $g_{-}^{\prime}$ is non-decreasing, left-continuous, and $g_{-}^{\prime}(x)$ is constant if $x>M$ or $x \leq-M$, hence $g_{-}^{\prime}$ is bounded on $\mathbb{R}$. The measure $\mu$, defined by $\mu([a, b)):=g_{-}^{\prime}(b)-g_{-}^{\prime}(a)$ if $a<b$, is a regular, finite, positive Borel measure on $\mathbb{R}$, with total mass $\mu(\mathbb{R})=g_{+}^{\prime}(M)-g_{-}^{\prime}(-M)$.

We are going to prove (13) pathwise. For this, let $\Omega_{0}, \mathbb{P}\left\{\Omega_{0}\right\}=1$, denote a subset of the sample space $\Omega$, on which, as $m \rightarrow \infty, B_{m}(t)$ uniformly converges to $W(t)$ on $[0, K]$ and $\mathcal{L}_{m}^{ \pm}(t, x)$ uniformly converges to $\mathcal{L}^{ \pm}(t, x)$ on $[0, K] \times \mathbb{R}$, cf. Theorem B and Corollary 1 above. We fix an $\omega \in \Omega_{0}$. Then, obviously, $W(t)$ and $\mathcal{L}^{ \pm}(t, x)$ will have continuous paths.

Consider the first term on the right side of (14). We want to show that it uniformly converges to $g(W(t))-g(W(0))$ for $t \in[0, K]$. With the notation $t_{m}:=\left\lfloor t 2^{2 m}\right\rfloor 2^{-2 m}$,

$$
\begin{aligned}
& \sup _{t \in[0, K]}\left|T_{x=a}^{B_{m}\left(t_{m}\right)} g_{-}^{\prime}(x) 2^{-m}-\int_{a}^{W(t)} g_{-}^{\prime}(x) \mathrm{d} x\right| \\
& \leq \sup _{t \in[0, K]}\left|T_{x=a}^{B_{m}\left(t_{m}\right)} g_{-}^{\prime}(x) 2^{-m}-\int_{a}^{B_{m}\left(t_{m}\right)} g_{-}^{\prime}(x) \mathrm{d} x\right| \\
& +\sup _{t \in[0, K]}\left|\int_{B_{m}\left(t_{m}\right)}^{W(t)} g_{-}^{\prime}(x) \mathrm{d} x\right| .
\end{aligned}
$$

The first term on the right side of 15 goes to zero as $m \rightarrow \infty$, because $g_{-}^{\prime}$ is non-decreasing, hence Riemann integrable on any compact interval $\left[a, B_{m}\left(t_{m}\right)\right]$, the trapezoidal sum is a Riemann sum on the same interval, so their difference can be estimated from above by the difference of the upper sum and the lower sum of $g_{-}^{\prime}$ on $\left[a, B_{m}\left(t_{m}\right)\right]$, which, in turn is dominated by $2^{-m}\left(g_{+}^{\prime}(M)-g_{-}^{\prime}(-M)\right)$ for any $t \in[0, K]$.

The second term on the right side of (15) converges to 0 as well, since $B_{m}\left(t_{m}\right)$ converges uniformly to $W(t)$ for $t \in[0, K]$, and $g_{-}^{\prime}$ is bounded on $\mathbb{R}$.

Since the slope of $g$ at any point is bounded above by $g_{+}^{\prime}(M)$ and below by $g_{-}^{\prime}(-M)$, we see that $g$ is Lipschitz, so absolutely continuous. Hence

$$
\int_{W(0)}^{W(t)} g_{-}^{\prime}(x) \mathrm{d} x=g(W(t))-g(W(0)) .
$$

Thus we have proved that

$$
\sup _{t \in[0, K]}\left|g(W(t))-g(W(0))-T_{x=a}^{B_{m}\left(\left\lfloor t 2^{2 m}\right\rfloor 2^{-2 m}\right)} g_{-}^{\prime}(x) 2^{-m}\right| \rightarrow 0
$$

almost surely as $m \rightarrow \infty$.

Now we turn to the convergence of the second term on the right side of (14). Fixing an $\omega \in \Omega_{0}$, we want to show that as $m \rightarrow \infty$,

$$
\sup _{t \in[0, K]}\left|\sum_{x \in a+2^{-m} \mathbb{Z}} \mathcal{L}_{m}^{+}(t, x)\left(g_{-}^{\prime}\left(x+2^{-m}\right)-g_{-}^{\prime}(x)\right)-\int_{-\infty}^{\infty} \frac{1}{2} \mathcal{L}(t, x) \mu(\mathrm{d} x)\right| \rightarrow 0
$$


(The other half containing the $\mathcal{L}_{m}^{-}(t, x)$ terms is similar.) Now, we have that

$$
\begin{aligned}
& \sup _{t \in[0, K]}\left|\sum_{x \in a+2^{-m} \mathbb{Z}} \mathcal{L}_{m}^{+}(t, x)\left(g_{-}^{\prime}\left(x+2^{-m}\right)-g_{-}^{\prime}(x)\right)-\int_{-\infty}^{\infty} \frac{1}{2} \mathcal{L}(t, x) \mu(\mathrm{d} x)\right| \\
& \leq \sup _{t \in[0, K]} \sum_{x \in a+2^{-m} \mathbb{Z}}\left|\mathcal{L}_{m}^{+}(t, x)-\frac{1}{2} \mathcal{L}(t, x)\right|\left(g_{-}^{\prime}\left(x+2^{-m}\right)-g_{-}^{\prime}(x)\right) \\
& +\frac{1}{2} \sup _{t \in[0, K]}\left|\sum_{x \in a+2^{-m} \mathbb{Z}} \mathcal{L}(t, x)\left(g_{-}^{\prime}\left(x+2^{-m}\right)-g_{-}^{\prime}(x)\right)-\int_{-\infty}^{\infty} \mathcal{L}(t, x) \mu(\mathrm{d} x)\right| .
\end{aligned}
$$

The first term on the right side of (18) goes to zero, since, by Corollary 1 . $\mathcal{L}_{m}^{+}(t, x)$ uniformly converges to $\frac{1}{2} \mathcal{L}(t, x)$ on $[0, K] \times \mathbb{R}$, and

$$
\sum_{x \in a+2^{-m} \mathbb{Z}}\left(g_{-}^{\prime}\left(x+2^{-m}\right)-g_{-}^{\prime}(x)\right) \leq g_{+}^{\prime}(M)-g_{-}^{\prime}(-M)<\infty .
$$

The second term on the right side of (18) also goes to zero, since it is the difference of a Riemann-Stieltjes integral and its approximating sum. Recall that $\mathcal{L}(t, x)$ is continuous, non-decreasing in $t$ for any $x, \mathcal{L}(K, x)$ has compact support, so bounded, as $x$ varies over $\mathbb{R}$, and the total mass $\mu(\mathbb{R})<\infty$.

Thus by (16) and (17), we have established the statements of the theorem for measures $\mu$ supported in a compact interval $[-M, M]$.

Consider now the case of a general $g$. Let $T^{M}:=\inf \{t>0:|W(t)| \geq M\}$ for $M=1,2, \ldots$, and approximate $g$ by

$$
g^{M}(x)= \begin{cases}g(M)+g_{+}^{\prime}(M)(x-M) & \text { if } x>M \\ g(x) & \text { if }|x| \leq M \\ g(-M)+g_{-}^{\prime}(-M)(x-M) & \text { if } x<-M\end{cases}
$$

Define $\mu^{M}$ as the measure determined by the change of $\left(g^{M}\right)_{-}^{\prime}$, which is supported in $[-M, M]$ with finite mass. Clearly, $g(W(t))=g^{M}(W(t))$ if $0 \leq$ $t \leq T^{M}$ and, since $\mathcal{L}\left(T^{M}, x\right)=0$ for all $x,|x|>M, \int \mathcal{L}(t, x) \mu(\mathrm{d} x)=$ $\int \mathcal{L}(t, x) \mu^{M}(\mathrm{~d} x)$ if $0 \leq t \leq T^{M}$.

The previous argument proves (12) for the interval $\left[0, K \wedge T^{M}\right]$, and also (13) if $0 \leq t \leq T^{M}$. Since the stopping times $\left(T^{M}\right)_{M=1}^{\infty}$ increase to $\infty$ a.s., this proves the theorem.

Comparing Itô's formula (11) and Itô-Tanaka formula (13) when $g$ is $C^{2}$ and convex, we obtain that

$$
\int_{0}^{t} g^{\prime \prime}(W(s)) \mathrm{d} s=\int_{-\infty}^{\infty} \mathcal{L}(t, x) g^{\prime \prime}(x) \mathrm{d} x .
$$

Since this holds for any continuous and positive function $g^{\prime \prime}$, by a monotone class argument we obtain the well-known occupation time formula for any bounded, Borel measurable function $h$ :

$$
\int_{0}^{t} h(W(s)) \mathrm{d} s=\int_{-\infty}^{\infty} h(x) \mathcal{L}(t, x) \mathrm{d} x
$$


As a special case, one gets $\int_{-\infty}^{\infty} \mathcal{L}(t, x) \mathrm{d} x=t$. Also, from (13) with $g(x)=$ $|x-a|$, one obtains Tanaka's formula

$$
\mathcal{L}(t, a)=|W(t)-a|-|W(0)-a|-\int_{0}^{t} \operatorname{sgn}(W(s)-a) \mathrm{d} W(s) .
$$

\section{Integration of predictable processes}

In this section our aim is to show that when the integrand $(Y(t))_{t \geq 0}$ is a predictable process satisfying some integrability condition, our previous approach to approximate the stochastic integral by sums of values of the integrand at the Skorohod stopping times $s_{m}(k)$, multiplied by $\pm 2^{-m}$, where the signs are obtained by a fair coin tossing sequence, still works. In other words, the standard non-random partitions of a time interval $[0, t]$ may be replaced by our random partitions $0=s_{m}(0)<s_{m}(1)<\cdots<s_{m}\left(\left\lfloor t 2^{2 m}\right\rfloor\right)$ of Skorohod embedding of random walks into the Wiener process. This is not surprising, because, as Lemma A shows, such Skorohod partitions are asymptotically equivalent to partitions $\left(k 2^{-2 m}\right)_{k=0}^{t 2^{2 m}}$, as $m \rightarrow \infty$. This approach will be described rather briefly, since in this case the details are essentially the same as in the standard approach to stochastic integration found in textbooks.

Let $\left(\mathcal{F}_{t}\right)_{t \geq 0}$ denote the natural filtration defined by our BM $W$. In this paper, stopping times and adapted processes are understood with respect to this filtration. By definition, we say that $Y$ is a simple, adapted process if there exist stopping times $0 \leq \tau_{0} \leq \tau_{1} \leq \cdots$ increasing to $\infty$ a.s. and random variables $\xi_{0}, \xi_{1}, \ldots$ such that $\xi_{j}$ is $\mathcal{F}_{\tau_{j}}$-measurable, $\mathbb{E}\left(\xi_{j}^{2}\right)<\infty$ for all $j$, and

$$
Y(t)=\xi_{0} \mathbf{1}_{\{0\}}(t)+\sum_{r=1}^{\infty} \xi_{r-1} \mathbf{1}_{\left(\tau_{r-1}, \tau_{r}\right]}(t) \quad(t \geq 0) .
$$

In the sequel, the only case we will consider is when, with a given $m \geq 0$, the stopping time sequence is the one given by the Skorohod embedding (3). Let $Y$ be a left-continuous, adapted process and with a fixed $b>0$, take the truncated process

$$
Y^{b}(t):=\left\{\begin{aligned}
b & \text { if } Y(t) \geq b \\
Y(t) & \text { if }|Y(t)|<b \\
-b & \text { if } Y(t) \leq-b
\end{aligned}\right.
$$

Further, with $m \geq 0$ fixed, take

$$
Y_{m}^{b}(t):=Y^{b}(0) \mathbf{1}_{\{0\}}(t)+\sum_{r=1}^{\infty} Y^{b}\left(s_{m}(r-1)\right) \mathbf{1}_{\left(s_{m}(r-1), s_{m}(r)\right]}(t) \quad(t \geq 0),
$$

with the Skorohod stopping times (3). Then $Y_{m}^{b}$ is a simple, adapted process.

Then, similarly as in (10), a stochastic sum of $Y_{m}^{b}$ is defined as

$$
\begin{aligned}
\left(Y_{m}^{b} \cdot W\right)_{t} & :=\sum_{r=1}^{\left\lfloor t 2^{2 m}\right\rfloor} Y^{b}\left(s_{m}(r-1)\right)\left(W\left(s_{m}(r)\right)-W\left(s_{m}(r-1)\right)\right) \\
& =\sum_{r=1}^{\left\lfloor t 2^{2 m}\right\rfloor} Y^{b}\left(s_{m}(r-1)\right) X_{m}(r) 2^{-m}
\end{aligned}
$$


where $\left(X_{m}(r)\right)_{r=1}^{\infty}$ is a sequence of independent, $\mathbb{P}\left\{X_{m}(r)= \pm 1\right\}=\frac{1}{2}$ random variables. Observe that (22) without the $X_{m}(r)= \pm 1$ factors would be asymptotically an ordinary Riemann sum of the integral $\int_{0}^{t} Y^{b}(s) \mathrm{d} s$. (The differences $s_{m}(r)-s_{m}(r-1)$ asymptotically equal $2^{-m}$ by Lemma A ) The random \pm 1 factors multiplying the terms of the sum transform it into an approximation of a stochastic integral.

It is clear that the usual properties of stochastic integrals hold for such stochastic sums. Namely, one has linearity: $\left(\alpha Y_{m}^{b}+\beta Z_{m}^{b}\right) \cdot W=\alpha Y_{m}^{b} \cdot W+\beta Z_{m}^{b}$. $W$, zero expectation: $\mathbb{E}\left(Y_{m}^{b} \cdot W\right)=0$, isometry: $\mathbb{E}\left(Y_{m}^{b} \cdot W\right)_{K}^{2}=\int_{0}^{K} \mathbb{E}\left(Y_{m}^{b}(t)\right)^{2} \mathrm{~d} t$, etc.

Lemma 2. Let $K>0$ be fixed and $Y$ be a left-continuous, adapted process such that

$$
\|Y\|_{K}^{2}:=\int_{0}^{K} \mathbb{E} Y^{2}(t) \mathrm{d} t<\infty .
$$

Then there exists a sequence $(m(b))_{b=1}^{\infty}$ such that $\left\|Y-Y_{m(b)}^{b}\right\|_{K} \rightarrow 0$ as $b \rightarrow \infty$, where $Y_{m}^{b}$ is defined by (21).

Proof. First, for any $b$,

$$
\begin{aligned}
\left\|Y-Y^{b}\right\|_{K}^{2} & \leq \int_{0}^{K} \mathbb{E}\left(Y^{2}(t) \mathbf{1}_{\{|Y(t)| \geq b\}}\right) \mathrm{d} t \\
& \leq \int_{0}^{K} \mathbb{E} Y^{2}(t) \mathrm{d} t<\infty .
\end{aligned}
$$

Therefore $\left\|Y-Y^{b}\right\|_{K} \rightarrow 0$ as $b \rightarrow \infty$ by dominated convergence.

Further, for $b$ fixed and $m \rightarrow \infty, Y_{m}^{b}(t) \rightarrow Y^{b}(t)$ a.s. for all $t \in[0, K]$, hence $\left\|Y^{b}-Y_{m}^{b}\right\|_{K} \rightarrow 0$ by bounded convergence. These prove the lemma.

So let $Y$ be a left-continuous, adapted process such that $\|Y\|_{K}<\infty$ and take $J_{b}(t):=\left(Y_{m(b)}^{b} \cdot W\right)_{t}, t \in[0, K]$, where $Y_{m(b)}^{b}$ is defined by the previous lemma. Since $Y_{m(b)}^{b}$ tends to $Y$ in $L^{2}([0, K] \times \Omega)$, by isometry, $J_{b}(t)$ tends to a random variable $J(t)$ in $L^{2}(\Omega)$, which is defined as the stochastic integral $\int_{0}^{t} Y(s) \mathrm{d} W(s)$ for $t \in[0, K]$.

From this point, the discussion of the usual properties of stochastic integrals and extensions to more general integrands largely agrees with the standard textbook case, therefore omitted.

\section{Extension to continuous local martingales}

The extension of the methods of the previous sections to continuous local martingales is rather straightforward. By a useful theorem of Dambis and DubinsSchwarz (DDS), a continuous local martingale $M$ can be transformed into a Brownian motion $W$ by a change of time, when time is measured by the quadratic variation $[M]_{t}$.

Let a right-continuous and complete filtration $\left(\mathcal{F}_{t}\right)_{t \geq 0}$ be given in $(\Omega, \mathcal{F}, \mathbb{P})$ and let the continuous local martingale $M$ be adapted to it. Define

$$
T_{s}=\inf \left\{t \geq 0:[M]_{t} \geq s\right\} \quad(s \geq 0) .
$$


Theorem D. If $M$ is a continuous $\left(\mathcal{F}_{t}\right)$-local martingale vanishing at 0 and such that $[M]_{\infty}=\infty$ a.s., then $W(s)=M\left(T_{s}\right)$ is an $\left(\mathcal{F}_{T_{s}}\right)$-Brownian motion and $M(t)=W\left([M]_{t}\right)$.

Similar statement is true when $[M]_{\infty}<\infty$ is possible, cf. [8, p. 182]. For example, $M(t)=W\left([M]_{t}\right)$ still holds for any $t \geq 0$.

Now some results are recalled from [11. Skorohod-type stopping times can be defined for $M$, similarly as for $W$ in (3). For $m \geq 0$, let $\tau_{m}(0)=0$ and

$$
\tau_{m}(k+1)=\inf \left\{t: t>\tau_{m}(k),\left|M(t)-M\left(\tau_{m}(k)\right)\right|=2^{-m}\right\} \quad(k \geq 0) .
$$

Then $s_{m}(k)=[M]_{\tau_{m}(k)}$ and $B_{m}\left(k 2^{-2 m}\right)=W\left(s_{m}(k)\right)=M\left(\tau_{m}(k)\right)$, where $s_{m}(k)$ is defined by (3) for the DDS BM $W$ and $B_{m}$ is a scaled simple, symmetric RW, as in previous sections.

Let $N_{m}(t)$ denote the following discrete quadratic variation process of $M$ :

$$
\begin{aligned}
N_{m}(t) & =2^{-2 m} \#\left\{r: r>0, \tau_{m}(r) \leq t\right\} \\
& =2^{-2 m} \#\left\{r: r>0, s_{m}(r) \leq[M]_{t}\right\} \quad(t \geq 0) .
\end{aligned}
$$

The next theorem is a reformulation of [11, Theorems 2 and 4], correcting some unfortunate errors there.

Theorem E. Let $K>0$ and take a sequence $\left(c_{m}\right)$ increasing to $\infty$ arbitrary slowly. Then for a.e. $\omega \in \Omega$, if $m \geq m_{0}(\omega)$, we have

$$
\sup _{0 \leq t \leq K}\left|[M]_{t}-N_{m}(t)\right|<c_{m} m^{\frac{1}{2}} 2^{-m}
$$

and

$$
\sup _{0 \leq t \leq K}\left|M(t)-B_{m}\left(N_{m}(t)\right)\right|<c_{m} m^{\frac{3}{4}} 2^{-\frac{m}{2}} .
$$

Thus $N_{m}(t)$ almost surely uniformly converges on compacts to $[M]_{t}$, and $B_{m}\left(N_{m}(t)\right)$ similarly converges to $M(t)$.

Combining the DDS time change and the pathwise approximations of local time described in Section 2, it is not too hard to generalize the local time results of 12 to continuous local martingales $M$. Let the $m$ th approximate local time of $M$ at a point $x \in 2^{-m} \mathbb{Z}$ up to time $t \in 2^{-2 m} \mathbb{Z}_{+}$be defined by

$$
\mathcal{L}_{m}^{M, \pm}(t, x)=2^{-m} \#\left\{s: B_{m}(s)=x, B_{m}\left(s+2^{-2 m}\right)=x \pm 2^{-m}\right\},
$$

where $s \in 2^{-2 m} \mathbb{Z} \cap\left[0,[M]_{t}\right)$ and $B_{m}\left(j 2^{-2 m}\right)=M\left(\tau_{m}(j)\right)$. Then $\mathcal{L}_{m}^{M, \pm}(t, x)$ can be extended to any $t \in \mathbb{R}_{+}$and $x \in \mathbb{R}$ by linear interpolation, as a continuous two-parameter process.

The major difference compared to Corollary 1 is that that the time interval $[0, K]$ has to be replaced by an interval of the form $\left[0, T_{b}^{K}\right]$ here, where $T_{b}^{K}:=$ $T_{b} \wedge K$, over which $[M]_{t}$ is bounded by a constant $b$.

Corollary 2. On any strip $\left[0, T_{b}^{K}\right] \times \mathbb{R}$, the approximate local times $\left(\mathcal{L}_{m}^{M,+}(t, x)\right)$ a.s. uniformly converge as $m \rightarrow \infty$ to the one half of the local time $\mathcal{L}^{M}(t, x)=$ $\mathcal{L}^{W}\left([M]_{t}, x\right)$, where $\mathcal{L}^{W}$ denotes the local time of the DDS BM of $M$. For any $C>1$, and for any $K>0, b \geq e$, and $m \geq 1$ such that $b 2^{2 m} \geq N(C)$, we have

$$
\begin{array}{r}
\mathbb{P}\left\{\sup _{(t, x) \in\left[0, T_{b}^{K}\right] \times \mathbb{R}}\left|\frac{1}{2} \mathcal{L}^{M}(t, x)-\mathcal{L}_{m+1}^{M,+}(t, x)\right| \geq 50 C b^{\frac{1}{4}}(\log b)^{\frac{3}{4}} m^{\frac{3}{4}} 2^{-\frac{m}{2}}\right\} \\
\leq \frac{32}{1-4^{1-C}}\left(b 2^{2 m}\right)^{1-C} .
\end{array}
$$


Similar statements hold for $\left(\mathcal{L}_{m}^{-}(t, x)\right)$ as well.

From this, by the Borel-Cantelli lemma, we get that for a.e. $\omega \in \Omega$, if $m \geq m_{0}(\omega)$, we have

$$
\begin{aligned}
& \sup _{(t, x) \in\left[0, T_{b}^{K}\right] \times \mathbb{R}}\left|\frac{1}{2} \mathcal{L}^{M}(t, x)-\mathcal{L}_{m+1}^{M,+}(t, x)\right| \\
& =\sup _{(t, x) \in[0, K] \times \mathbb{R}}\left|\frac{1}{2} \mathcal{L}^{M}\left(t \wedge T_{b}^{K}, x\right)-\mathcal{L}_{m+1}^{M,+}\left(t \wedge T_{b}^{K}, x\right)\right| \\
& \leq 50 C b^{\frac{1}{4}}(\log b)^{\frac{3}{4}} m^{\frac{3}{4}} 2^{-\frac{m}{2}} .
\end{aligned}
$$

If we replace $b$ here by a sequence $\left(c_{m}\right)$ increasing to $\infty$, then, by the continuity of $[M]_{t}, T_{c_{m}} \nearrow \infty$, and so $t \wedge T_{c_{m}}^{K} \nearrow t$ for $t \in[0, K]$. Hence we have

Corollary 3. Let $K>0$ and take a sequence $\left(c_{m}\right)$ increasing to $\infty$ arbitrary slowly. Then for a.e. $\omega \in \Omega$, if $m \geq m_{0}(\omega)$, we have

$$
\sup _{(t, x) \in[0, K] \times \mathbb{R}}\left|\frac{1}{2} \mathcal{L}^{M}(t, x)-\mathcal{L}_{m}^{M, \pm}(t, x)\right|<c_{m} m^{\frac{3}{4}} 2^{-\frac{m}{2}} .
$$

Next define Itô integrals w.r.t. $M$ when the integrand is a 'nice' function of $M$. An Itô sum is defined by

$$
\begin{aligned}
(f(M) \cdot M)_{t}^{m} & :=\sum_{\tau_{m}(r) \leq t} f\left(M\left(\tau_{m}(r-1)\right)\right) 2^{-m} X_{m}(r) \\
& =\sum_{s_{m}(r) \leq[M]_{t}} f\left(W\left(s_{m}(r-1)\right)\right) 2^{-m} X_{m}(r),
\end{aligned}
$$

where $2^{-m} X_{m}(r)=M\left(\tau_{m}(r)\right)-M\left(\tau_{m}(r-1)\right)=W\left(s_{m}(r)\right)-W\left(s_{m}(r-1)\right)$, $\left(X_{m}(r)\right)_{r=1}^{\infty}$ being an independent, \pm 1 symmetric coin tossing sequence.

Theorem 2. Let $g$ be a $C^{2}$-function, alternatively, be the difference of two convex functions. Then for arbitrary $K>0$,

$$
\sup _{t \in[0, K]}\left|\left(g_{-}^{\prime}(M) \cdot M\right)_{t}^{m}-\int_{0}^{t} g_{-}^{\prime}(M(s)) \mathrm{d} M(s)\right| \rightarrow 0
$$

almost surely as $m \rightarrow \infty$, and in the first case one gets the Itô formula

$$
g(M(t))-g(M(0))=\int_{0}^{t} g^{\prime}(M(s)) \mathrm{d} M(s)+\frac{1}{2} \int_{0}^{t} g^{\prime \prime}(M(s)) \mathrm{d}[M]_{s} ;
$$

in the second case one obtains the Itô-Tanaka formula

$$
g(M(t))-g(M(0))=\int_{0}^{t} g_{-}^{\prime}(M(s)) \mathrm{d} M(s)+\frac{1}{2} \int_{-\infty}^{\infty} \mathcal{L}^{M}(t, x) \mu(\mathrm{d} x) .
$$

The proof is similar to the Brownian case, therefore omitted.

(23) indicates that if the function $f$ is $C^{1}$ or of finite variation on bounded intervals, then

$$
\int_{0}^{t} f(M(s)) \mathrm{d} M(s)=\int_{0}^{[M]_{t}} f(W(s)) \mathrm{d} W(s) .
$$


We mention that the DDS BM $W$ and the quadratic variation $[M]$ are independent processes if and only if $M$ is symmetrically evolving (is an Ocone martingale) in the sense that given the past of $M$, distributions of future increments are invariant under reflection, cf. [11, Theorem 5].

Finally, we can extend the method of integration of predictable integrands discussed in Section 4 to integrals w.r.t. a continuous local martingale $M$. Let $K>0$ be fixed and $Y$ be a left-continuous, adapted process on $[0, K]$ such that

$$
\|Y\|_{M, K}^{2}:=\mathbb{E} \int_{0}^{K} Y^{2}(t) \mathrm{d}[M]_{t}<\infty .
$$

For $b>0$ let $Y^{b}$ denote the truncated process (20). If $m \geq 0$ fixed, take

$$
Y_{m}^{b}(t):=Y^{b}(0) \mathbf{1}_{\{0\}}(t)+\sum_{r=1}^{\infty} Y^{b}\left(\tau_{m}(r-1)\right) \mathbf{1}_{\left(\tau_{m}(r-1), \tau_{m}(r)\right]}(t)
$$

for $t \geq 0$. Then $Y_{m}^{b}$ is a simple, adapted process.

An Itô sum of $Y_{m}^{b}$ is defined as

$$
\begin{aligned}
\left(Y_{m}^{b} \cdot M\right)_{t} & :=\sum_{\tau_{m}(r) \leq t} Y^{b}\left(\tau_{m}(r-1)\right)\left(M\left(\tau_{m}(r)\right)-M\left(\tau_{m}(r-1)\right)\right) \\
& =\sum_{\tau_{m}(r) \leq t} Y^{b}\left(\tau_{m}(r-1)\right) X_{m}(r) 2^{-m}
\end{aligned}
$$

where $\left(X_{m}(r)\right)_{r=1}^{\infty}$ is a sequence of independent, $\mathbb{P}\left\{X_{m}(r)= \pm 1\right\}=\frac{1}{2}$ random variables.

Here too, cf. Lemma 2, there exists a sequence $(m(b))_{b=1}^{\infty}$ such that $\| Y-$ $Y_{m(b)}^{b} \|_{M, K} \rightarrow 0$ as $b \rightarrow \infty$, where $Y_{m}^{b}$ is defined by (24). So take $J_{b}(t):=$ $\left(Y_{m(b)}^{b} \cdot M\right)_{t}, t \in[0, K]$. Since $Y_{m(b)}^{b}$ tends to $Y$ in $L_{M}^{2}([0, K] \times \Omega)$, by isometry, $J_{b}(t)$ tends to a random variable $J(t)$ in $L^{2}(\Omega)$, which is defined as the stochastic integral $\int_{0}^{t} Y(s) \mathrm{d} M(s)$ for $t \in[0, K]$.

\section{References}

[1] Akahori, J. (2005) A discrete Ito calculus approach to He's framework for multi-factor discrete market. Asia Pacific Financial Markets 12, 273-287.

[2] Csörgö, M. and Révész, P. (1985) On the stability of the local time of a symmetric random walk. Acta Sci. Math. 48, 85-96.

[3] Fujita, T. (2003) A random walk analogue of Lévy's theorem. Preprint, Hitotsubashi University.

[4] Karandikar, L.R. (1995) On pathwise stochastic integration. Stoch. Proc. Appl. 57, 11-18.

[5] Knight, F.B. (1962) On the random walk and Brownian motion. Trans. Amer. Math. Soc. 103, 218-228.

[6] Kudzma, R. (1982) Ito's formula for a random walk. Litovskii Matematicheskii Sbornik 22, 122-127. 
[7] Révész, P. (1981) Local times and invariance. In: Analytic Methods in Probability Theory. Lecture Notes in Math. No. 861. pp. 128-145. Springer, Berlin.

[8] Revuz, D. and Yor, M. (1999) Continuous Martingales and Brownian Motion. Third edition, Springer, Berlin.

[9] Szabados, T. (1990). A discrete Itô's formula. Limit Theorems in Probability and Statistics (Pécs, 1989), Colloq. Math. Soc. János Bolyai, 57, NorthHolland, Amsterdam, 491-502.

[10] Szabados, T. (1996) An elementary introduction to the Wiener process and stochastic integrals. Studia Sci. Math. Hung. 31, 249-297.

[11] Székely, B. and Szabados, T. (2004) Strong approximation of continuous local martingales by simple random walks. Studia Sci. Math. Hung. 41, 101-126.

[12] Szabados, T. and Székely, B. (2005) An elementary approach to Brownian local time based on simple, symmetric random walks. Period. Math. Hung. 51, 79-98. 